\title{
Optimal Channel Allocation Algorithm with Efficient Bandwidth Reservation for Cellular Networks
}

\author{
K. Madhavi \\ Asst. Professor \\ JNTUA College of Engg \\ Anantapur, AP, India
}

\author{
K. Sandhya Rani \\ Professor \\ Dept. of Computer Science \\ SPMVV, Tirupathi, AP, India
}

\author{
P. Chandrasekhar Reddy \\ Professor \\ JNTUH College of Engg, \\ Hyderabad, India
}

\begin{abstract}
As the growth of mobile users increasing in the present scenario and because of limited bandwidth available, there is a need to efficiently use the bandwidth available. The quality of service can be maximized by efficient bandwidth reservation. In this paper, the cross layer based bandwidth reservation scheme is proposed which initially reserves some amount of bandwidth for handoff flows. After that the bandwidth can be increased for handoff flows by the base station based on the user mobility. The user may not only go straight but also left, right and backwards. This paper considers all possibilities of user movements and bandwidth is reserved accordingly. Therefore making the base stations to dynamically increase the reserved bandwidth for handoffs when the initially reserved bandwidth is insufficient reduces the end to end delay and increases the throughput of the system. The proposed system performance is compared with the legacy systems and is shown to be better.
\end{abstract}

\section{General Terms}

Mobile Computing, Cellular Networks, Bandwidth etc

\section{Keywords}

Hand offs, Channel allocation, QoS etc.

\section{INTRODUCTION}

In cellular wireless networks, the challenges are increasing more and more as there are encroachments in the technology. Optimization leads to many more challenges to dig out more from the scientific growth [11].

The enormous growth of mobile users in the present scenario with limited bandwidth is a challenging task today. The bandwidth should be utilized in an optimal way so that more number of users may be serviced. One of the important factors to improve the quality of cellular service is to make handoffs nearly invisible to the user and successful [5]. Unsuccessful handoff requests are one of the main causes of end to end delay.

In spite of the last several years of research on wireless ad-hoc networks, massive real-life deployments of ad-hoc networks still remain a challenge. Although the freedom of ad-hoc networks from utilizing fixed infrastructure for offering wireless communication services makes them attractive for fast deployment in application domains such as the military and emergency services, they are limited by their ability to efficiently offer global accessibility and web-based services such as file sharing, messenger services and voice-over-IP [12]. Nodes of traditional cellular wireless networks are maintained by a base station manager (BSM) or server for routing. On the other hand, nodes of purely ad-hoc networks behave as routers by relaying messages in order to improve the performance of the network. One of the most important issues in providing ubiquitous communication is mobility management [13], which primarily concern effectively tracking the locations of the nodes. In case of hybrid networks, BSM can be used for effective mobility management, which can be otherwise more challenging in ad-hoc networks, because of their lack in using a dedicated router/server having a network-wide knowledge of the location of the nodes. In the case of pure cellular wireless networks, the goal of adaptive call admission control is to ensure that there is sufficient bandwidth reservation for handoff, i.e., for transferring an ongoing call in a cell to another. The reserved bandwidth in a target cell is proportional to the traffic intensity in the surrounding cells [3]. In the absence of sufficient bandwidth for handoff, new connections are subject to getting dropped. One common approach used to reduce the connection dropping rate (CDR) is to reserve some bandwidth solely for handoff use [4]. [1]

In a cellular network, a mobile user may visit different cells in his lifetime. In each of these cells, resources must be made available to support the mobile user else the user will suffer a forced termination of his call in progress. Therefore, careful resource allocation along with call admission control is required to mitigate the chances of forced termination or dropping of a call. Keeping the probability of a user getting dropped (Pdrop) below a pre-specified target value is considered as a practical design goal of any resource allocation scheme. Achieving the above goal provides the probablistic quality of service (QoS) guarantee as desired by a mobile user. [9]

Early work in handoff prioritization proposes the static reservation of bandwidth at each BS as a solution [14], in which a fixed portion of the radio capacity is permanently reserved for handoffs. However, such a static approach is unable to handle variable traffic load and mobility [15]. [10]

\section{BACKGROUND}

Bandwidth reservation is an important issue to improve the performance of cellular networks. There are proposals for bandwidth reservation for both cellular networks and ad-hoc networks, where we present some of the relevant pieces of work.

In the case of pure cellular wireless networks, the goal of adaptive call admission control is to ensure that there is sufficient bandwidth reservation for handoff, i.e., for transferring an ongoing call in a cell to another. The reserved bandwidth in a target cell is proportional to the traffic intensity in the surrounding cells [3]. In the absence of sufficient 
bandwidth for handoff, new connections are subject to getting dropped. One common approach used to reduce the connection dropping rate (CDR) is to reserve some bandwidth solely for handoff use [4].

A cross-layer-based QoS model is proposed in [6] to categorize various flows for service differentiation as well as reservation. The primary focus of this work is to reserve the bandwidth for real-time and non-real-time (best effort) flows. The proposed architecture in [1] aims to provide CLIASM [6] to the network layer and to its lower and higher layers. The network layer collects the information from the application layer and forms the ad-hoc network. The ad-hoc network is further classified into hierarchical regions based on mobility, and then these regions are mapped to the BSM of a cellular network.

An efficient Hash Table-Based Node Identification (HTNI) Method using which bandwidth for various flows can be reserved is proposed in [1]. Bandwidth reservation depends on the type of the traffic and its priorities. Bandwidth reservation factor is defined for use in hybrid network environments. A cross-layer-based architecture for bandwidth reservation is proposed to maintain Quality-of-Service (QoS). A priority reallocation method for flows which starve for long time is done in [1].

An algorithm for channel allocation is proposed in [2] which uses the system model where channels are reserved for originating calls, handoff calls separately and some channels are left free which can be used by both originating and handoff calls. The system is modeled using Markov model. The channel allocation procedure is done based on distributed dynamic allocation procedure. Reusability concept is used and the channels are divided into different groups. The groups are allocated to BS's based on mutual exclusion paradigm. The QoS parameters like blocking probability and dropping probability is examined.

\section{SYSTEM MODEL}

In the Bandwidth reservation based on user mobility scheme, we assume that base stations are equipped with road-map information and that mobile stations are equipped with global positioning systems (GPS) devices. Mobile stations periodically report their GPS location information to their base stations. Based on the location information of the mobile stations at two consecutive epochs, the base stations estimate the speed and moving direction of the mobile stations. Furthermore, the base stations estimate the probability that the mobile stations will enter the neighboring cells based on their velocity and the roadmap information stored in the base stations. The base stations then compute the amount of bandwidth to be reserved, based on such estimation. With the road-map information, the base stations can make a more-accurate prediction on the user's mobility and, hence, reduce unnecessary bandwidth reservation. The structure of the cellular network estimated is shown in Fig. 1.

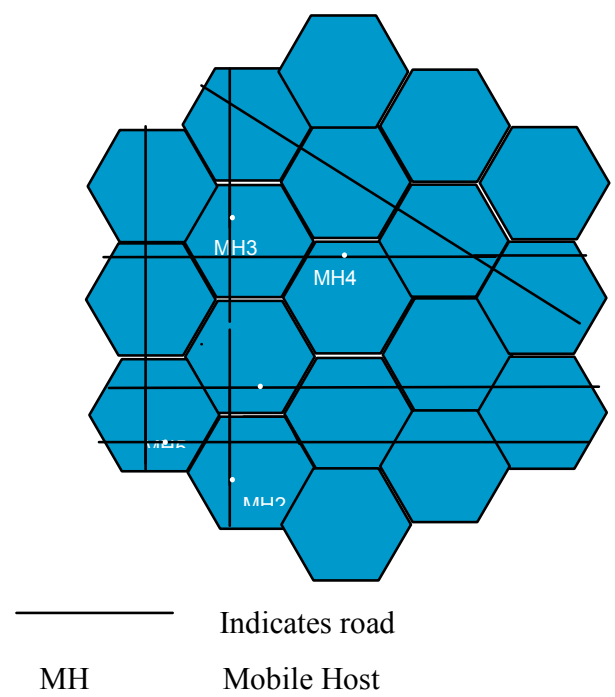

Fig.1: Structure of Cellular Network

Mobile user's motion pattern is mostly restricted by man-made constructions, such as roads. Obviously, mobile users who are making handoff requests are in motion; most are either walking or riding in automobiles on the roads. That is to say, mobility is always restricted by the layout of the roads. A mobile user's mobility is restricted in the sense that mobile users can only move on the roads. We propose a new mobility prediction and bandwidth-reservation method called "bandwidth reservation based on user mobility". This scheme makes use of a mobile user's moving speed, direction, and the road information stored in the base stations to predict the handoff probabilities to neighboring cells. The amount of reserved bandwidth is dynamically adjusted according to the handoff probability and the traffic load in each cell.

\subsection{Measuring Traffic Intensity to Know How Many Channels to Reserve at Each Base Station}

The intensity of the traffic varies during the day. It is normally high in morning business hours and less in afternoon during lunchtime and again goes high in evening. The traffic in busiest periods are called busy-hour traffic. The intensity of the busyhour traffic again varies also depending on the day of the week. During weekends traffic in morning time may be less and evening time will be high. The network operator needs to meet the demands of the average busy-hour traffic.

Traffic intensity can be measured using two dimensionless units

$$
\text { 1.) Erlang }
$$

\section{2.) Circuit Centum Seconds (CCS)}

One Erlang is equivalent to number of calls (made in one hour) multiplied by the duration of these calls (in hours). Each call has a different duration or a different call holding time: for traffic intensity measurements the average call holding time is taken into account[7][8]. The typical values for average call holding time vary between 120 and 180 seconds. Therefore, the traffic intensity in Erlangs can be defined as: 
$\mathrm{T}$ (inErlangs) $=$ Number of call in an hour $*$ average call holding time in $\mathrm{sec} / 3600$

If a call attempt is made when all channels in cellular networks are serving other calls, the call attempt will be blocked. The probability of call blocking in a telecommunication network is call Grade of Service (GoS). The Grade of Service of a telecommunication network varies between zero and one. A GoS of 0.02 is normally taken as acceptable for communication systems.

\subsection{Estimating the Number of Subscribers in the Cellular System}

The number of subscribers in the system can be estimated assuming the relation between the number of subscribers in the busy hour (n) and the number of calls per hour per cell. The maximum number of calls per hour that a cell can take depends on the number of channels allocated for that cell based on traffic conditions under its geographic area. The estimated number of subscribers in the system $M$ is

$\mathrm{M}=\sum$ maximum number of calls per cell $/ \mathrm{n}$

In this paper, we consider the flows to be as originating and handoff flows. The originating flows are the flows which originated in a particular cell and the handoff flows are generated when a mobile user moves from one cell location to another. The cross layer architecture proposed in [6] is been used for bandwidth reservation scheme proposed in this paper. We also use the architecture proposed in [1]. In this architecture, the call admission control estimates the available bandwidth and reserves the same for handoff flows. In this paper, we propose the bandwidth reservation scheme which reserves some amount of bandwidth for handoff flows and some amount of bandwidth for originating flows and keeps some amount of bandwidth which can be used by both handoff flows and originating flows [2].

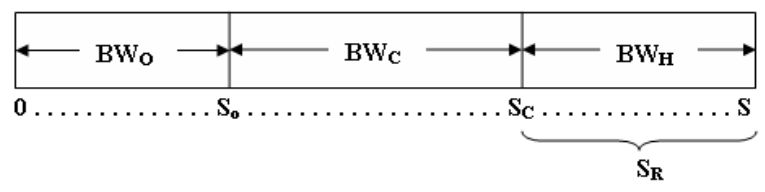

Fig. 2 System Model

The proposed system model is shown in Fig. 2. In Fig. 2, BW represents the bandwidth reserved for originating flows, $\mathrm{BW}_{\mathrm{C}}$ represents the bandwidth which can be utilized by both originating and handoff flows and $\mathrm{BW}_{\mathrm{H}}$ represents the bandwidth which can be utilized by only handoff flows. The bandwidth procedure defined in this paper is fair-fair reservation which maintains the minimum delay and provide high throughput. To allocate the bandwidth for originating flow, the $\mathrm{BW}_{\mathrm{O}}$ region is verified. If bandwidth can be allocated, it is done otherwise the $\mathrm{BW}_{\mathrm{C}}$ is checked and bandwidth is allocated. Finally if the bandwidth cannot be allocated the originating flow is blocked. To allocate the bandwidth for handoff flow, the $\mathrm{BW}_{\mathrm{H}}$ region is verified. If bandwidth can be allocated, it is done otherwise the $\mathrm{BW}_{\mathrm{C}}$ is checked and bandwidth is allocated. Finally if the bandwidth cannot be allocated the handoff flow is dropped. This procedure is explained below.

\subsection{Bandwidth Reservation Procedure}

1. For originating flow

a. if bandwidth can be allocated from BWO then

i. bandwidth is allocated from BWO

b. else

i. if bandwidth can be allocated from BWC then

1. bandwidth is allocated from BWC

ii. else

1. call is blocked

2. else

3. For handoff flow

a. if bandwidth can be allocated from BWH then

i. bandwidth is allocated from BWH

b. else

if bandwidth can be allocated from BWC then

bandwidth is allocated from BWC

else

call is dropped

\section{PERFORMANCE ANALYSIS}

The performance of the proposed system is estimated using the following metrics:

- Packet Delivery Throughput:

The ratio of the number of packets received by the destinations to the number of packets sent by the CBR sources is defined as packet delivery throughput.

- End-to-End Delay of Data Packets:

The difference between the time at which the packet is received by the destination and the time at which the packet is sent by the source is defined as end to end delay of data packets. The packets lost in the journey from source to destination are not considered. The delay metric do not includes the delay related to the route discovery, queuing and retransmissions.

The proposed system performance is compared with the RSVP and EDCF reservation policies. Fig. 3 and Fig. 4 shows the performance results of comparison in terms of throughput and delay respectively and can be observed that the performance is 
improved by the proposed system with respect to the legacy systems.

The QoS parameters specified above are examined by simulation. Analytically they are examined by using the blocking and dropping probabilities formulae [2] given below.

The blocking probability for an originating call is given as

$B_{O}=\sum_{i=S_{C}+1}^{S} P(i)$

The dropping probability for a handoff call is given as

$B_{H}=\frac{\left(\lambda_{O}+\lambda_{H}\right)^{S_{C}-S_{O}} \lambda_{H}^{S-S_{C}}}{S ! \mu^{S}} P(0)$

Where,

$S=$ Total Bandwidth

$S_{R}=$ Bandwidth reserved for handoff flows.

$S_{O}=$ Bandwidth reserved for only originating calls

The steady state probability $\mathrm{P}(\mathrm{i})$ can be obtained:

$$
P(i)= \begin{cases}\frac{\left(\lambda_{0}^{i}\right)}{i ! \mu^{i}} P(0) & 0 \leq i \leq S_{O} \\ \frac{\left(\lambda_{O}+\lambda_{H}\right)^{i-S_{O}} \lambda_{O}^{S_{O}}}{i ! \mu^{i}} P(0) & S_{O}<i \leq S_{C} \\ \frac{\left(\lambda_{O}+\lambda_{H}\right)^{S_{C}-S_{O}} \lambda_{H}^{i-S_{C}}}{i ! \mu^{i}} P(0) & S_{C}<i \leq S\end{cases}
$$

Where,

$$
P(0)=\left[\begin{array}{c}
\sum_{i=0}^{S_{O}} \frac{\lambda_{O}^{i}}{i ! \mu^{i}}+\sum_{i=S_{O}+1}^{S_{C}} \frac{\left(\lambda_{O}+\lambda_{H}\right)^{i-S_{O}} \lambda_{O}^{S_{O}}}{i ! \mu^{i}}+ \\
\sum_{i=S_{C}+1}^{S} \frac{\left(\lambda_{O}+\lambda_{H}\right)^{S_{C}-S_{O}} \lambda_{O}^{S_{O}} \lambda_{H}^{i-S_{C}}}{i ! \mu^{i}}
\end{array}\right]^{-1}
$$

\subsection{Simulation Results}

The performance of the proposed system is evaluated by comparing the performance of the proposed system with the performance of RSVP and EDCF reservation policies. Fig. 3 shows the comparison of the performance of the proposed system to the RSVP and EDCF in terms of throughput and can be observed that the throughput is high for the proposed system.
The experiment is carried out for 35 runs for various flows between the number of flows and the number of packets transmitted successfully. It is observed that the throughput of the proposed system improves when compared with the performance of RSVP, EDCF methods. Fig. 4 shows the comparison of the performance of the proposed system to the RSVP and EDCF in terms of end to end delay of data packets and can be observed that the delay is low for the proposed system. The experiment is carried out for 35 runs for various flows between the number of flows and the time taken to successfully deliver the total flows. It is observed that the delay of the proposed system is low when compared with the performance of RSVP, EDCF methods.

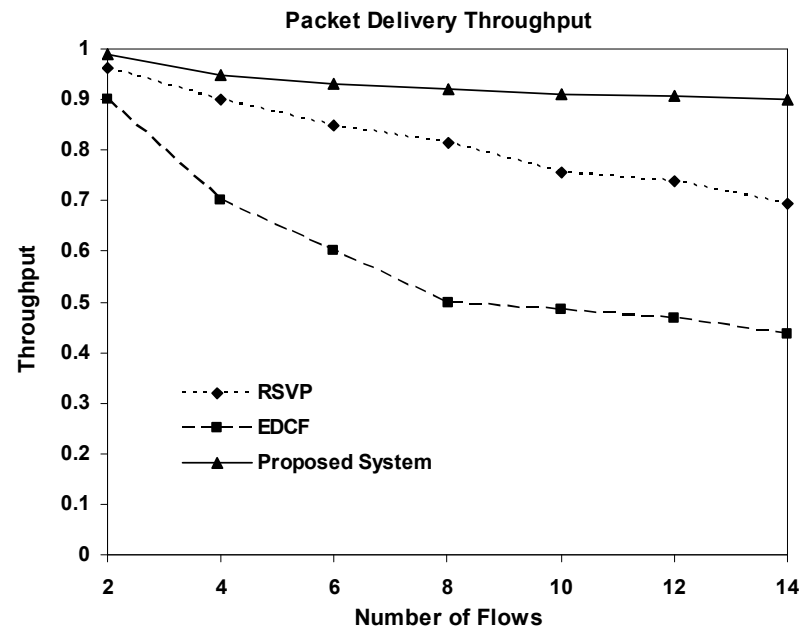

Fig. 3 Comparison of packet delivery throughput

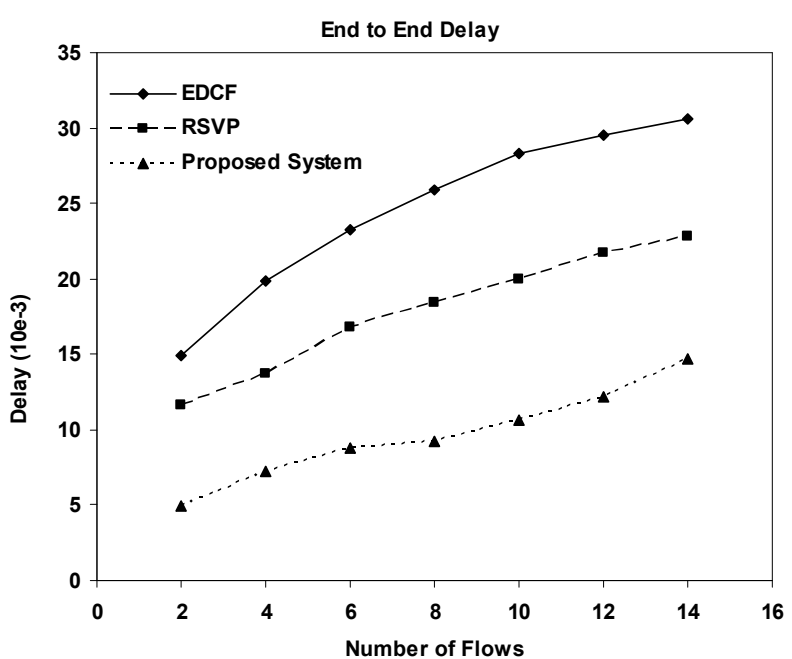

Fig. 4 Comparison of End to End Delay

\section{CONCLUSIONS}

A new efficient cross layer based bandwidth reservation scheme is proposed in this paper. The amount of bandwidth to be 
reserved at each base station was calculated dynamically based on the user mobility and the traffic intensity of mobile users. This paper assumes several probabilities that the user may move left, right, straight and backwards. The analysis of the QoS performance metrics like packet throughput and end to end packet delay is carried out and observed that the proposed system is better in performance in terms of delay and throughput metrics.

\section{ACKNOWLEDGMENTS}

Our thanks to the experts who have contributed towards development of this paper.

\section{REFERENCES}

[1] P. V. Krishna, N. C. S. N. Iyengar and S. Misra, "An Efficient Hash Table-Based Node Identification Method for Bandwidth Reservation in Hybrid Cellular and Ad-Hoc Networks", Computer Communications (Elsevier), Vol. 31, No. 4, 2008, pp. 722-733

[2] P. V. Krishna and N. Ch. S. N. Iyengar, 'Optimal Channel Allocation Algorithm with Efficient Channel Reservation for Cellular Networks', Int. J. Communication Networks and Distributed Systems, Vol. 1, No. 1, 2008, pp.33-51

[3] J. Misic, T.Y. Bun, On call level QoS guarantees under heterogeneous user mobilities in wireless multimedia networks, in: Proceedings of the IEEE Global Telecommunications Conference - GLOBECOM'99, Rio De Janerio, Brazil, 1999, pp. 2730-2736.

[4] S. Lim, G. Cao, C.R. Das, A differential bandwidth reservation policy for multimedia wireless networks, in: Proceedings of 2001 International Conference on Parallel Processing Workshops, Valencia, Spain, 2001, pp. 447.

[5] I. Katzela and M. Naghshineh, " Channel Assignment Schemes for Cellular Mobile Telecommunication Systems: a Comprehensive Survey," IEEE Personal Communications, Vol. 3, No. 3, pp. 10-31, June 1996.
[6] P. Venkata Krishna, N.Ch.S.N. Iyengar, A cross layer based QoS model for wireless and mobile networks, Journal of Mobile Communications 1 (4) (2007) 114-120.

[7] Paul Bedell, Wireless Crash Course (New York: Tata McGraw-Hill, 2002)

[8] Vijay K.Garg and Joseph E. Wilkes, Principles \& Applications of GSM (India: Pearson Education, 2005)

[9] Samrat Ganguly Badri Nath Navin Goyal, "Optimal Bandwidth Reservation Schedule in Cellular Networks", IEEE, 2003

[10] Wee-Seng Soh and Hyong S. Kim, "Dynamic Bandwidth Reservation in Cellular Networks using Road Topology based Mobility Prediction",

[11] Mohammad Anbar and D P Vidyarthi, "On Demand Bandwidth Reservation for Real-Time Traffic in Cellular IP Network using Particle Swarm Optimization", International Journal of Business Data Communications and Networking, Volume 5, Issue 3, 53-66, July September 2009

[12] Y.J. Song, D. Park, Infrastructure support for ad-hoc networks: when and where, International Journal of High Performance Computing and Networking 4 (1/2) (2006) 78-84.

[13] K.Y. Eng, M.J. Karol, M. Veeraraghavan, E. Ayanoglu, C.B. Woodworth, P. Pancha, R.A. Valenzuel, A wireless broadband adhoc ATM local-area network, IEEE Wireless Networks (1995) 161-174.

[14] D. Hong and S. S. Rappaport, "Traffic model and performance analysis for cellular mobile radio telephone systems with prioritized and nonprioritized handoff procedures," IEEE Trans. Veh. Technol., vol. VT-35, no. 3, Aug. 1986, pp. 77-92.

[15] S. Choi and K. G. Shin, "Predictive and adaptive bandwidth reservation for handoffs in QoS-sensitive cellular networks," in Proc. ACM SIGCOMM' 98, Vancouver, British Columbia, Sep. 1998, pp. 155-66. 